\title{
A study on backstreaming positive ions on a high power negative ion source for fusion
}

\author{
L. Schiesko, C. Hopf, P. Franzen, W. Kraus, R. Riedl, U. Fantz \\ and NNBI Team \\ Max-Planck-Institut für Plasmaphysik, EURATOM Association, Boltzmannstrasse 2, \\ D-85748 Garching, Germany \\ E-mail: loic.schiesko@ipp.mpg.de
}

\begin{abstract}
Future magnetic-confinement nuclear fusion experiments will be using neutral beam injection (NBI) systems for plasma heating that are based on sources for negative hydrogen ions as opposed to the positive ions mostly used to date. An unavoidable effect in negative NBI (NNBI) sytems is the creation of positive ions in the acceleration region due to collisions between the fast negative ions and the neutral background gas. These positive ions are accelerated back into the ion source. At the high extracted ion current densities from NNBI sources and the high acceleration voltages - $1 \mathrm{MeV}$ in the case of ITER - the resulting heat load on the backplate of the source and the sputtering rates of the backplate material can be substantial. In this work, sputtering probes and a simple 1D calculation were employed to estimate the flux density of the backstreaming ions in the rear part of the ion source at the long pulse NNBI testbed MANITU ( $20 \mathrm{kV}$ extraction voltage). It was found that the flux of backstreaming ions is approximately between 0.8 and $2.5 \%$ of the flux of negative ions extracted from the source. Experiment and theory are in fair agreement.
\end{abstract}

\section{Introduction}

Neutral beam injection heating [1] in nuclear magnetic-confinement fusion experiments typically produces fast neutrals by the neutralization of a fast hydrogen $(\mathrm{H}$ or $\mathrm{D})$ ion beam passing through hydrogen background gas. When starting from positive ions, the required reaction is charge transfer from the background gas to the moving ions. The efficiency of this neutralization reaction, however, decreases with relative velocity and becomes smaller than $50 \%$ above $\mathrm{D}^{+}$energies of about $100 \mathrm{keV}$. Larger fusion experiments in the future will require higher beam energies. For example the International Thermonuclear Experimental Reactor (ITER) will require $16 \mathrm{MW}$ of neutral beam power at up to $1 \mathrm{MeV}$ particle energy [2]. At these high energies only the neutralisation of negative hydrogen ions by collisions with background gas provides good yields. As a side effect, collisions between the fast particles and the background gas in the ion acceleration region also lead to the formation of positive ions which will be accelerated in the opposite direction into the ion source. With 
the high product of extracted negative ion current density and acceleration voltage, the power load and material erosion due to sputtering on the backplate of the source because of these backstreaming positive ions can be substantial. Fubiani estimated the total power transmitted back into the negative ion source (by $\mathrm{D}^{+}$and $\mathrm{D}_{2}^{+}$ions) to be $880 \mathrm{~kW}$ [3]. Such high power could cause damage of the backplate of the ITER ion source. In this study and for the first time, sputter probes and a simple 1dimensional calculation were used to estimate the flux of backstreaming ions in an NNBI testbed. Other techniques were already employed to determine the backstreaming ion flux: de Esch et al. [4] used a calorimeter to measure the power deposited by the ions. Umeda et al. [5] observed the the aperture pattern on the backplate of the source.

In 2007 the IPP RF source was chosen as the reference design for the plasma generation in the ITER NBI system. Three NNBI testbeds are currently operated at IPP. The BATMAN testbed is well diagnosed and dedicated to the understanding of negative ion generation and extraction $[6,7]$. The MANITU testbed aims at demonstrating that the ITER requirements can be achieved in long pulse operation (typically $3600 \mathrm{~s}$ ) [8, 9]. RADI is devoted to demonstrating the homogeneity and scalability of a large RF source $[10,11]$. A new testbed, ELISE, is under construction $[12,13]$. ELISE will be a "half size ITER source" and an intermediate step between the present IPP sources (1/8 ITER size) and the full ITER size.

Besides an interest in determining the flux and energy of backstreaming ions, there was also a practical interest to locate copper sources in IPP's long pulse testbed MANITU. Copper is the base material of the source. It has been reported earlier that after long pulse operation at MANITU, all inner surfaces were covered by a thin layer of copper [8]. Moreover, plasma spectroscopy showed copper lines. Initially, most of the copper was sputtered from the Faraday shield that is installed to protect the alumina insulator from plasma erosion. It was suspected that copper pollution affected the work function of the plasma grid where negative hydrogen ions are generated by the conversion of neutral and ionized hydrogen species, and hence reduced the source performance. In the presence of oxygen the sputtered copper impurity chemically reacts with the Cs layer. As a remedy, almost all the inner surfaces were then coated by a $3 \mu \mathrm{m}$ molybdenum layer. A great impact on source performance was found: a stabilisation of the co-extracted electron current at a lower level was observed as well as an increase of the extracted negative ion current [8]. A factor of ten in the decrease of the copper lines was observed with spectroscopy, indicating that the main source of copper had been the Faraday shield, but also that further sources were still present.

In order to identify the remaining copper sources in MANITU as well as to quantify the flux of backstreaming ions, sputter probes were distributed inside the source during two experimental campaigns. The probes carried thin strips of copper or chromium on a lighter base material. The thickness of the thin metal strips on the probes was determined by Rutherford backscattering spectroscopy (RBS) before and after exposure in the ion source. The experimental results were compared to a simple one-dimensional 


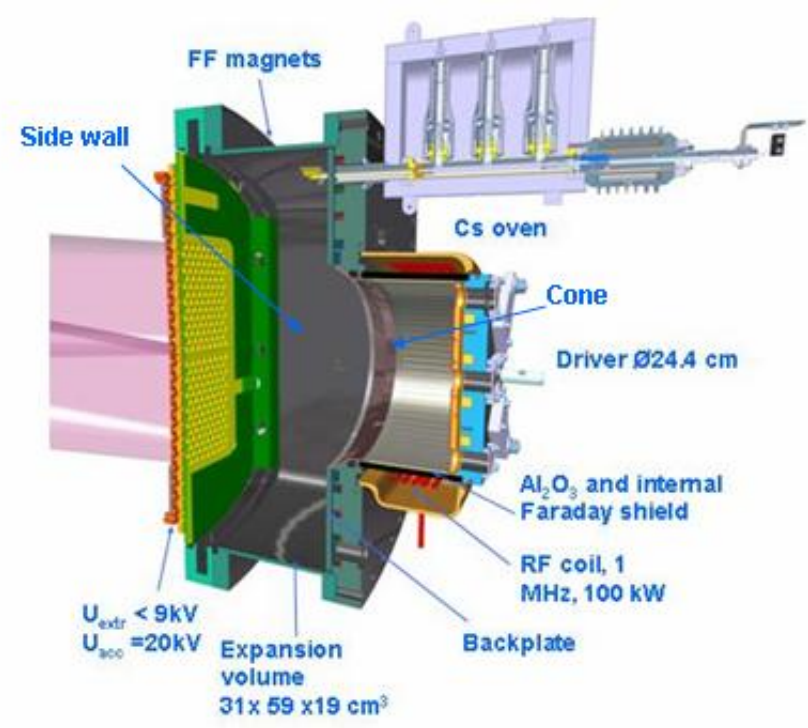

Figure 1. The IPP prototype source.

calculation.

This paper is organized as follows: the first part is devoted to the experimental setup, the second to the $1 \mathrm{D}$ model, while the the third part describes the experimental results and compares the results with calculations.

\section{Experimental setup}

The experiments were performed at the MANITU testbed. The source, shown in figure 1 , consists of three different regions. The plasma is generated in the driver, where the power $\left(P_{\max }=150 \mathrm{~kW}, f=1 \mathrm{MHz}\right)$ is coupled to the plasma by a water-cooled rf coil. The ceramic insulator is protected from the plasma by a copper Faraday shield coated with molybdenum. The plasma then diffuses into the expansion chamber and finally reaches the extraction region. A magnetic filter field with a flux density around $8 \mathrm{mT}$ separates the latter two parts of the source. The goal of the filter field is to reduce the electron temperature in order to minimize the destruction of the negative ions generated on the plasma grid (PG) surface and to reduce the amount of co-extracted electrons. Optimum source performance, i.e. high extracted negative ion current and low coextracted electron current, is achieved when caesium is evaporated into the source [14]. 
Typically, the evaporation rate is of the order of $10 \mathrm{mg} / \mathrm{h}$.

The extraction system consists of three grids: the plasma grid with chamfered apertures, the extraction grid and the grounded grid. The source is biased to a high negative potential, typically $-20 \mathrm{kV}$. The plasma grid temperature has to be in the range of $100-200^{\circ} \mathrm{C}$ for optimum performance. In order to deflect the co-extracted electrons out of the beam, the extraction grid hosts permanents magnets. The procedure to measure the electrical currents and especially to separate the contribution of the electrons from the contribution of the ions is described in [15].

In order to quantify the material erosion caused by the backstreaming ions, sputter probes were installed in the ion source during two experimental campaigns. The probes consisted of thin metal strips sputter-deposited on a substrate. The initial and final thickness of the strips was measured by Rutherford backscattering (RBS) analysis at a scattering angle of $165^{\circ}$ using $3 \mathrm{MeV}{ }^{4} \mathrm{He}$ as projectiles. The RBS measurements primarily yielded areal densities (atoms $/\left(\mathrm{cm}^{2} \mathrm{~s}\right)$ ) which were converted into thicknesses using the densities of the metals. Figure $2 \mathrm{a}$ is an example of the probes used during the second campaign, while figure $2 \mathrm{~b}$ shows the picture of a molten probe taken at the end of the second campaign.

The probes were placed in the remaining potential copper source locations. These were the uncoated $\mathrm{Cu}$ surfaces of the expansion chamber side walls and of the cone separating the driver from the expansion chamber. The side walls are only exposed to the outstreaming plasma with low energy while the cone is also exposed to the backstreaming ions with an energy sufficient for physical sputtering. For the first campaign four probes were used which consisted of a copper layer sputter-deposited on stainless steel. The thickness of the copper layer was $440 \mathrm{~nm}$. Three of the probes were located at the side walls and one was mounted on the cone. Unfortunately, the analysis of the probe from the cone after exposure showed that all copper had been eroded; hence, only a lower limit could be derived for the backstreaming ion flux.

The complete erosion during the first campaign led to a change of the design of the probes for the second campaign. The thickness of the $\mathrm{Cu}$ strip was increased to $(12.8 \pm 0.1) \times 10^{18} \mathrm{~cm}^{-2}(1505 \pm 12 \mathrm{~nm})$. Additionally, a second strip of chromium was deposited next to the copper with a thickness of $(5.5 \pm 0.1) \times 10^{18} \mathrm{~cm}^{-2}(625 \pm 12 \mathrm{~nm})$. The reason to choose a second material was a concern regarding the high reactivity of $\mathrm{Cu}$ with Cs. After the first campaign there was an indication that on some probes, the caesium on the surface had reacted with water after venting the source and the resulting $\mathrm{CsOH}$ had chemically attacked the copper. Chromium was chosen because it reacts less with $\mathrm{CsOH}$ and because its sputtering yield by hydrogen is very similar to that of copper. In order to achieve a better separation between copper/chromium and the substrate material in the RBS spectra, especially considering the increased thickness of the strips, the stainless steel substrate in the first campain was replaced by titanium. For better RBS resolution the titanium substrates were polished prior to deposition. Table 1 summarises the main ion source and sputter probe parameters.

In the second campaign eight of these new probes were symmetrically distributed on the 
A study on backstreaming positive ions on a high power negative ion source for fusion5

\begin{tabular}{|c|c|c|c|c|c|c|}
\hline & $\mathrm{Cr}(\mathrm{nm})$ & $\mathrm{Cu}(\mathrm{nm})$ & $p(\mathrm{~Pa})$ & $P(\mathrm{~kW})$ & $j_{\mathrm{H}^{-}}\left(\mathrm{mA} \mathrm{cm}^{-2}\right)$ & $U_{\text {acc }}(\mathrm{kV})$ \\
\hline $1^{\text {st }}$ camp. & & 440 & $0.4-0.6$ & $50-80$ & 14.5 & 25 \\
\hline $2^{\text {nd }}$ camp. & 652 & 1505 & $0.4-0.6$ & $50-80$ & 16.1 & 20 \\
\hline
\end{tabular}

Table 1. Probe and source parameters. $\mathrm{Cr}$ and $\mathrm{Cu}$ represents the deposited chromium and copper layer thickness, $p$ and $P$ the discharge pressure and power, $j_{\mathrm{H}^{-}}$the electrically measured negative ion current density and $U_{\text {acc }}$ the acceleration voltage between plasma grid and grounded grid.

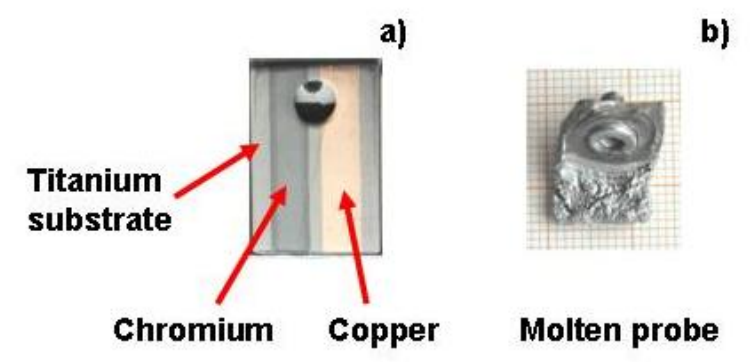

Figure 2. Photographs of an unexposed sputter probe as used during the second campaign (a) and of a molten probe after the second campaign (b). The dimensions of the probes are $1 \mathrm{~cm} \times 0.5 \mathrm{~cm}$.

cone of the driver (see Fig. 3) and one was installed on the side wall of the expansion chamber. The photograph in Fig. 3 was taken after the opening of the source at the end of the second campaign. One can see that some of the probes were partially or completely molten, which will be discussed in the experimental results section.

\section{A simple 1D calculation}

A simple one-dimensional calculation is used to estimate the flux and the energy distribution function of the two backstreaming ion species $\left(\mathrm{H}^{+}\right.$and $\left.\mathrm{H}_{2}^{+}\right)$. It is assumed that all the ions have an ideal linear trajectory as they follow the electrical field lines. Moreover, no ion-ion or ion-wall interaction is taken into account. Also, the negative ion beam attenuation due to various loss mechanisms is neglected.

The extraction of negative hydrogen ions inevitably leads to the simultaneous extraction of electrons from the ion source. These electrons are magnetically deflected onto the extraction grid to avoid their acceleration to full energy. They can ionize background neutrals, but the ionization cross section decreases rapidly with energy as can be seen in [16]. Hence, only reactions between the negative ions and the background gas as well as subsequent reactions of the products have to be taken into account.

Figure 4 shows the cross sections from [17] of the relevant reactions that generate backstreaming ions. The reactions are summarised in table 2. Reaction (1) is the double stripping of a fast $\mathrm{H}^{-}$, directly producing a fast $\mathrm{H}^{+}$, and reaction (2) is the simple stripping of a fast $\mathrm{H}^{-}$, producing a fast $\mathrm{H}^{0}$. The fast neutral from reaction (2) can in turn generate a slow positive ion via reaction (3b). Another possibility is the 
A study on backstreaming positive ions on a high power negative ion source for fusion6

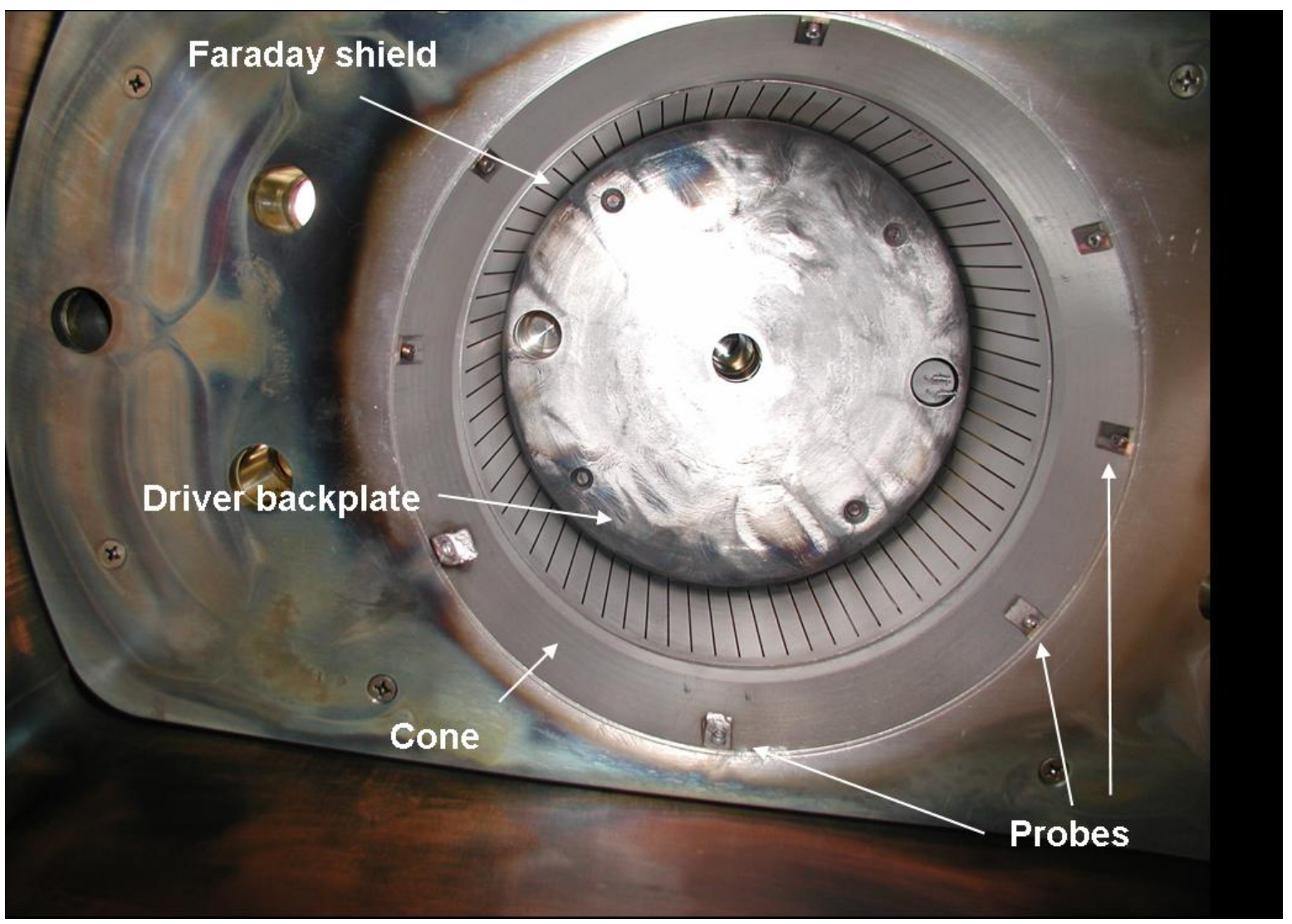

Figure 3. Photograph of the rear part of the source with the probes installed. The picture was taken after the end of the second campaign.

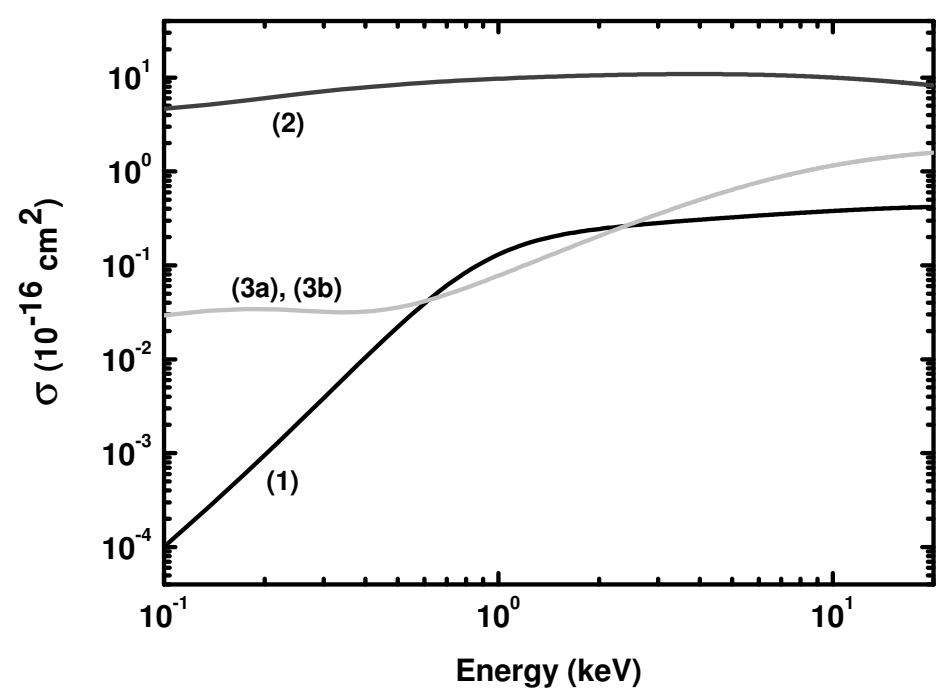

Figure 4. Cross sections of the different involved mechanisms (labels refers to reactions in table 1$)$. 
A study on backstreaming positive ions on a high power negative ion source for fusion 7

\begin{tabular}{lcc}
\hline $\begin{array}{l}\text { Reaction } \\
\text { number }\end{array}$ & \multicolumn{1}{c}{ Process } & Label \\
\hline 1 & $\mathrm{H}^{-}+\mathrm{H}_{2} \rightarrow \mathrm{H}^{+}$(fast) $+2 \mathrm{e}^{-}+\mathrm{H}_{2}$ & Double stripping \\
2 & $\mathrm{H}^{-}+\mathrm{H}_{2} \rightarrow \mathrm{H}^{0}$ (fast) $+\mathrm{e}^{-}+\mathrm{H}_{2}$ & Simple stripping \\
$3 \mathrm{a}$ & $\mathrm{H}^{-}+\mathrm{H}_{2} \rightarrow \mathrm{H}_{2}^{+}$(slow) $+2 \mathrm{e}^{-}+\mathrm{H}^{0}$ & Ionization \\
$3 \mathrm{~b}$ & $\mathrm{H}^{0}+\mathrm{H}_{2} \rightarrow \mathrm{H}_{2}^{+}$(slow) $+\mathrm{e}^{-}+\mathrm{H}^{0}$ & Ionization \\
\hline
\end{tabular}

Table 2. Main processes involved in the generation of backstreaming ions.

generation of a fast positive ion via the channel $\mathrm{H}^{0}+\mathrm{H}_{2} \rightarrow \mathrm{H}^{+}$(fast) $+\mathrm{e}^{-}+\mathrm{H}_{2}$. However, this channel is neglected since the reaction rate is low compared to the others. Reaction (3a) is the ionization of a background gas neutral directly by a negative ion. Reactions (1) and (3a) are single step processes and reaction (2) followed by (3b) is a two-step process. The cross section for the ionization of $\mathrm{H}_{2}$ by $\mathrm{H}^{-}$(reaction 3a) is equal to that of the ionization by $\mathrm{H}^{0}$ (reaction $3 \mathrm{~b}$ ) for energies below $50 \mathrm{keV}$ [18].

All reactions involving background atomic hydrogen are neglected as its density in the extraction system is significantly below that of $\mathrm{H}_{2}$. Spectroscopy measurements performed in front of the plasma grid showed an $\mathrm{H} / \mathrm{H}_{2}$ ratio of $2 \times 10^{-1}$ [20]. This ratio will be further reduced in the extraction system by recombination on the grid surfaces. The electrostatic potential in the extraction/acceleration system is shown in figure 5a. For the purpose of this calculation the initial energy of the negative ions in the plasma is negligible. The negative ions are accelerated by the voltage between the plasma grid (PG) and the extraction grid (EG) of typically $7.5 \mathrm{kV}$. Further acceleration occurs between the EG and grounded grid (GG). The voltage between EG and GG is typically $12.5 \mathrm{kV}$, resulting in a final energy of $20 \mathrm{keV}$.

Also shown in figure $5 \mathrm{a}$ is the pressure profile between the PG and GG for $0.4 \mathrm{~Pa}$ source pressure, which is close to the ITER required source pressure. This profile was calculated from the source pressure and the conductances of the grid apertures. This calculation was also tried for the ITER NBI extraction system and the resulting pressure profile agrees within $20 \%$ with that of a detailed Monte Carlo model [19]. During the second campaign the discharge pressure was varied between 0.4 and $0.6 \mathrm{~Pa}$. In order to keep the calculation simple only the profiles for the two extremes of $0.4 \mathrm{~Pa}$ and $0.6 \mathrm{~Pa}$ were calculated. To calculate the positive ion production rates the background gas density profile is required. To calculate this from the pressure profile the gas temperature profile is needed. Optical emission spectroscopy showed that the neutral gas temperature is of the order of $1200 \mathrm{~K}$ [21] in front of the PG. Temperature accommodation is assumed to occur at the entrance of the extraction aperture as shown in Fig. 5b. The resulting density variation within the acceleration zone calculated for $0.4 \mathrm{~Pa}$ source pressure is also presented in figure 5b. The increase in density between PG and EG is a result of the assumed decrease of the background gas temperature.

For the single-step processes in table 2 the local birth rate $\Gamma$ of positive ions in an interval $\Delta x\left(\Delta x=1.87 \times 10^{-2} \mathrm{~mm}\right)$ around position $x$ is proportional to the product $n(x) \sigma \Delta x$, 

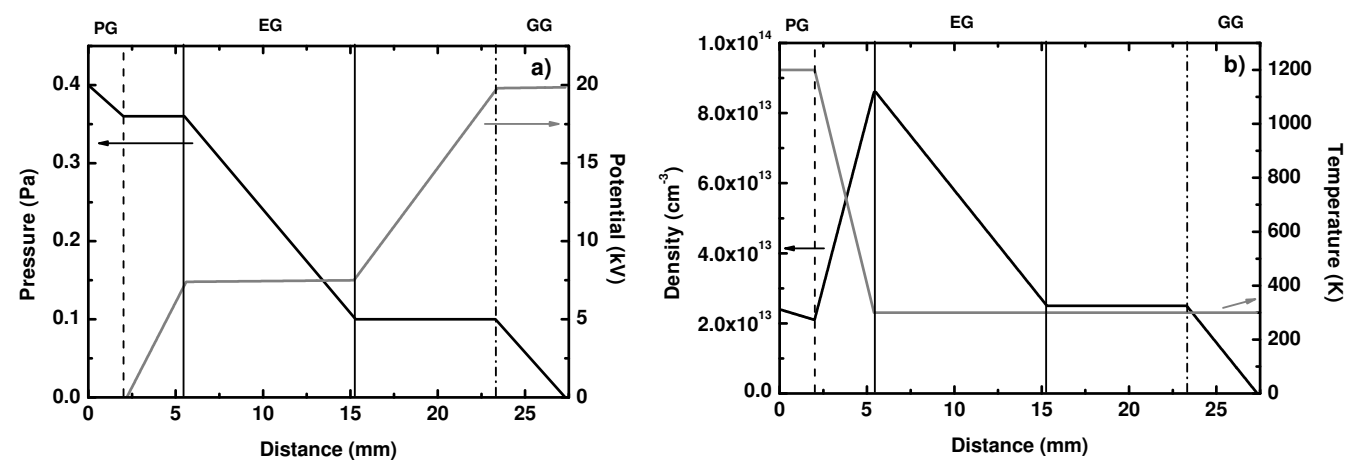

Figure 5. a) Pressure and electrostatic potential in the accelerator. b) Density and background gas temperature. The dimensions correspond to the large area grid.

with $n$ being the background gas density and $\sigma$ the cross section, as long as beam attenuation is negligible. For the two-step processes, starting from a fast $\mathrm{H}^{0}$, the local birth rate is

$$
\gamma=j n(x) \sigma_{2} \Delta x \int_{0}^{x} \mathrm{~d} \xi n(\xi) \sigma_{1}
$$

where $\sigma_{1}$ and $\sigma_{2}$ are the cross sections for the first and second reaction step and $j$ the negative ion flux density. The product $n \sigma \Delta x$ is shown in figure 6a. Table 3 lists the fractions of the positive ions created in different parts of the accelerator. It appears that most of the backstreaming ions are created within the extraction grid.

The energy distribution functions for $\mathrm{H}^{+}$and $\mathrm{H}_{2}^{+}$were calculated from the total local birth rates of theses species, $\Gamma\left(\mathrm{H}^{+}\right)$and $\Gamma\left(\mathrm{H}_{2}^{+}\right)$. As the $\mathrm{H}_{2}^{+}$ions are created at rest the IEDF is given by

$$
f\left(E, \mathrm{H}_{2}^{+}\right)=\Gamma(x(E)) \cdot\left(\frac{\mathrm{d} V}{\mathrm{~d} x}\right)^{-1}
$$

where $E$ is the energy of a negative ion at position $x$. The $\mathrm{H}^{+}$ions are not created at rest but with the kinetic energy of the original fast $\mathrm{H}^{-}$or $\mathrm{H}^{0}$ from which they were created. For the double stripping reaction (1) this means that an $\mathrm{H}^{+}$created at location $x_{0}$ will have an initial kinetic energy of $e V\left(x_{0}\right)$ in $+x$ direction, if $V(x)$ is the electrostatic potential. The ion will then be decelerated in the increasing potential, invert its direction and be accelerated again in $-x$ direction. When reaching the location $x_{0}$ again, its kinetic energy is again $e V\left(x_{0}\right)$, but this time in $-x$ direction. On its way from $x=x_{0}$ to $x=0$ it will gain another amount $\mathrm{eV}\left(x_{0}\right)$ of kinetic energy, hence its final energy will be $2 e V\left(x_{0}\right)=2 E\left(x_{0}\right)$. Thus the IEDF in this case is given by

$$
f\left(E, \mathrm{H}^{+}\right)=\Gamma(x(E / 2)) \cdot\left(\frac{\mathrm{d} V}{\mathrm{~d} x}\right)^{-1} .
$$


A study on backstreaming positive ions on a high power negative ion source for fusion 9

\begin{tabular}{|c|c|c|c|}
\hline & $\mathrm{PG} \leq \mathrm{x} \leq \mathrm{EG}$ & $\mathrm{EG}$ & $\mathrm{EG} \leq \mathrm{x} \leq \mathrm{GG}$ \\
\hline Strip. & $20.1 \%$ & $55.8 \%$ & $24.1 \%$ \\
\hline $\mathrm{H}^{+}$ & $15.5 \%$ & $53.1 \%$ & $31.4 \%$ \\
\hline $\mathrm{H}_{2}^{+}$ & $10.3 \%$ & $52.3 \%$ & $37.4 \%$ \\
\hline
\end{tabular}

Table 3. Relative contribution of the different zones of the extraction system to positive ion generation.
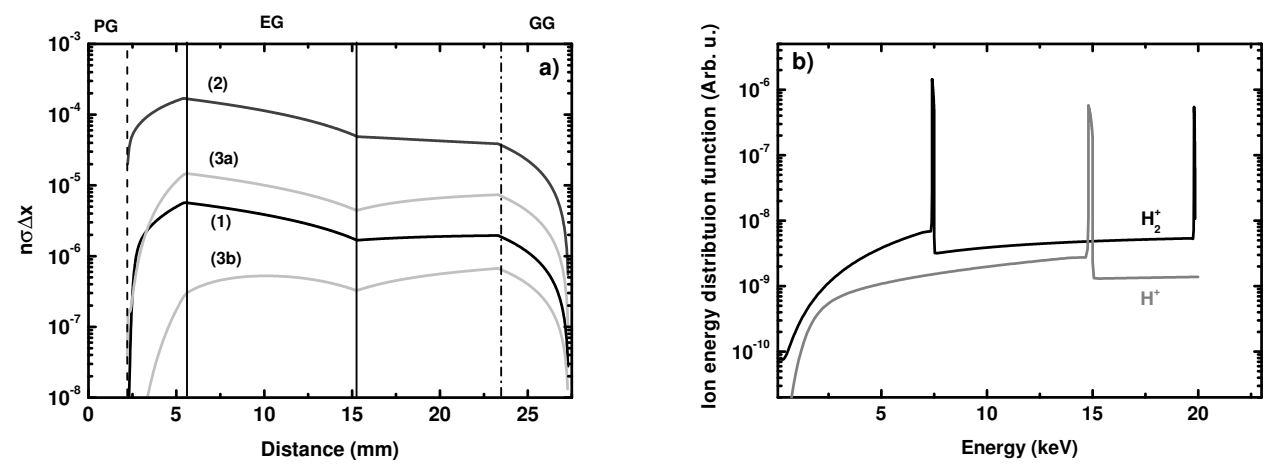

Figure 6. a) Local positive ion birth rate. b) Backstreaming ion energy distribution function.
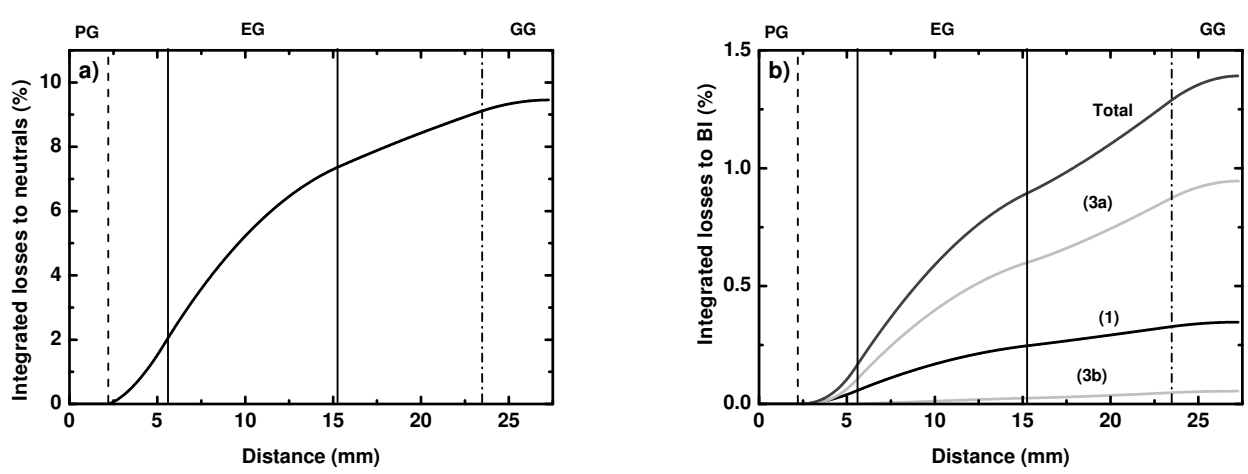

Figure 7. a) Integrated losses to neutrals. b) Integrated losses to backstreaming ions for each contributing mechanism.

Figure $6 \mathrm{~b}$ shows the resulting ion energy distribution functions for the $\mathrm{H}^{+}$and $\mathrm{H}_{2}^{+}$ions. A proportion of $36 \%$ of $\mathrm{H}^{+}$and $64 \%$ of $\mathrm{H}_{2}^{+}$ions was found. The two peaks at $7.5 \mathrm{keV}$ and $20 \mathrm{keV}$ in the $\mathrm{H}_{2}^{+}$IEDF correspond to the backstreaming ions generated at the almost constant potentials within the extraction grid and within the grounded grid, 
respectively (see figure 5a). The $\mathrm{H}^{+}$ion distribution function has only one peak at 15 $\mathrm{keV}$, i.e. twice the energy corresponding to the potential of the EG. A second peak does not appear because ions created at potentials above $10 \mathrm{kV}$ cannot be completely decelerated and hence escape in $+x$ direction. The height and the width of the peaks depends of the exact assumptions on field penetration into the grid holes, i. e. by how much the potential varies within the grids. However, as the cross sections in Fig. 4 change slowly with energy the integral under the peaks is conserved independent of these detailed assumptions.

Figure 7a represents the losses to simple stripping while figure $7 \mathrm{~b}$ shows the losses $L$ to positive ions for all the involved mechanisms,

$$
L=1-\exp \left(-\int_{0}^{x} \mathrm{~d} \xi n(\xi) \sigma(\xi)\right) .
$$

At $0.4 \mathrm{~Pa}, 9.5 \%$ of the negative ion beam is lost due to neutralization by simple stripping and $1.4 \%$ is lost due to all reactions that create backstreaming ions. The total beam loss is $11 \%$. The estimated loss due to simple stripping is thus greater than what was measured at MANITU (3\% at $0.4 \mathrm{~Pa})$ [22]. Assuming a higher temperature in the extraction aperture than the $300 \mathrm{~K}$ chosen for the calculation leads to a much better fit to the experimental data. However, the justification for assuming this higher temperature is not clear up to now. It is unlikely that no accommodation in the extraction gap occurs, however beam heating of the gas in the accelerator was observed at JET NBI [23].

\section{Experimental results}

After both campaigns, an initial visual inspection of the sputter probes showed that the shadow of the head of the screw holding the probe in place could be clearly seen. The orientation and position of this shadow - precisely behind the screw with respect to the direction of the backstreaming ion flux — proves that it is caused by backstreaming ions. The contrast between the shadowed and non-shadowed part is a consequence of two competing processes, deposition of caesium and of various sputtered wall materials from other wall locations, and the local sputtering due to the backstreaming ions. Whereas the flux of depositing species is more or less isotropic and causes no sharp shadow, the backstreaming ions have a well-defined direction.

During the first campaign, all the copper of the probe located on the cone was eroded. The total exposure time to backstreaming ions was $48000 \mathrm{~s}$. Consequently, only a lower limit of the backstreaming flux could be determined. Expressed as a fraction of the flux of extracted negative ions - from here onwards denoted as "backstreaming ion fraction" - this lower limit with error is between 2.2 and $2.7 \%$. The evaluation procedure will be detailed for the second campaign. None of the probes located at the side walls of the expansion chamber showed any erosion.

After the second campaign, RBS analysis showed that of the six probes which were located in the flux of the backstreaming ions, two were completely molten and one 
partially, rendering further analysis impossible. The remaining three probes showed alloy formation at the $\mathrm{Cu} / \mathrm{Ti}$ and $\mathrm{Cr} / \mathrm{Ti}$ interfaces at varying degrees, also indicating that they had experienced high temperatures for extended periods of time; the total exposure time of the samples was $86000 \mathrm{~s}$. Bad heat contact between the probe and the cone surface in combination with the comparatively low heat conductivity of $\mathrm{Ti}$ and long pulse operation (up to several minutes) must have been responsible for the high temperatures. Some of the probes on which alloy formation was found also showed evidence of sputtering, but quantitative analysis was hampered by the strong mixing of all materials due to the alloy formation.

Finally, only one of the non-molten probes showed low enough alloy formation not to hamper the analysis. The erosion was determined to be $(10.3 \pm 0.1) \times 10^{18}$ atoms cm $^{-2}$

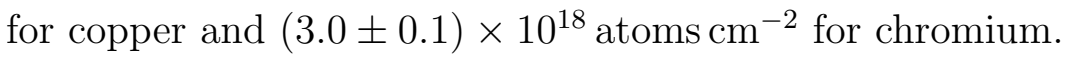

In order to derive the flux of the backstreaming ions from these numbers one needs to know the mean sputtering yield. In a first step, the energy-dependent sputtering yields $Y(E)$ for $\mathrm{H}^{+} \rightarrow \mathrm{Cu}$ and $\mathrm{H}^{+} \rightarrow$ Cr were calculated with the TRIM.SP code [24]. In these calculations an angle of incidence with respect to the surface normal of $45^{\circ}$ was assumed as is the case for the samples mounted on the cone. The surface binding energies were taken from Eckstein et al. [25], who report 3.52 and $4.12 \mathrm{eV}$ for $\mathrm{Cu}$ and $\mathrm{Cr}$, respectively. In order to calculate the mean sputtering yield the energy distribution function, $f(E)$, of the backstreaming ions needs to be known. It was obtained by the $1 \mathrm{D}$ calculation described in the previous section. The mean yield can then be computed by

$$
\langle Y\rangle=\alpha \int \mathrm{d} E f\left(E, \mathrm{H}^{+}\right) Y(E)+2 \beta \int \mathrm{d} E f\left(E, \mathrm{H}_{2}^{+}\right) Y(E / 2)
$$

where

$$
\int \mathrm{d} E f(E)=1 \text { and } \alpha+\beta=1
$$

In this relation, $\langle Y\rangle$ is the mean yield, $f\left(E, \mathrm{H}^{+}\right)$and $f\left(E, \mathrm{H}_{2}^{+}\right)$are the calculated ion energy distribution functions of $\mathrm{H}^{+}$and $\mathrm{H}_{2}^{+}$ions, respectively. For $\mathrm{H}_{2}^{+}$it is assumed that upon hitting the surface the molecule ion breaks up and the situation is hence equivalent to the impact of two $\mathrm{H}$ atoms at half the energy. $\alpha$ and $\beta$ are the fractions of backstreaming ion flux carried by $\mathrm{H}^{+}$and $\mathrm{H}_{2}^{+}$.

As mentioned earlier, the height and width of the peaks in the IEDFs depend on the assumed field penetration into the grid holes while the integral over the peaks is largely unaffected. As the energy-dependent sputtering yield can always be locally approximated by a linear dependence, the mean sputtering yield in Eq. (5) is also very insensitve to the peak width.

It should be noted that the analysis of the sputter probes assumes that the flux of backstreaming ions is homogeneous within the exposed part of the backplate and cone. If this was not the case, i. e. if the beamlets of backstreaming ions were strongly focussed [3], one should visually see an image of the pattern of extraction holes on the backplate after long operation. MANITU has been running with the same driver backplate for several years and no such pattern was seen. Hence, it is assumed that the beamlets of 
backs treaming ions are sightly divergent and overlapping at the backplate, leading to a constant flux density in the exposed region of the rear part of the ion source.

Using the eroded number of atoms together with the mean yields, the following incident flux densities of backstreaming ions are obtained:

- $2.5 \times 10^{15} \mathrm{~cm}^{-2} \mathrm{~s}^{-1}$ from copper erosion and

- $7.6 \times 10^{14} \mathrm{~cm}^{-2} \mathrm{~s}^{-1}$ from chromium erosion.

With a mean extracted negative ion current density of $16.1 \mathrm{~mA} \mathrm{~cm}^{-2}$ these numbers correspond to backstreaming ion fractions of $2.5 \%$ and $0.8 \%$ for copper and chromium erosion, respectively. As a reminder, the lower limit estimated from the first campaign was $2.2 \%$.

The reason for the apparent difference observed between the adjacent copper and chromium strips is still an open question. One possible explanation might be that the flux of the backstreaming ions is not as homogeneous at the backplate as we have assumed, solely based on visual inspection of the backplate. A second possible explanation may be the previously discussed chemical reactions of Cs with copper. If this is true, the result obtained on chromium is more accurate than that obtained on copper. Other possible explanations include chemical surface reactions between the metals and impurities in the source which might alter the surface binding energy or dilute the density of target atoms of the surface. Such effects on the sputtering yield are for instance known for oxide formation on metals [26]. The influence of target dilution due to the deposition of other elements, most notably molybdenum sputtered from Mo-coated surfaces, was estimated based on the deposition of these elements found in the shadow of the screw. For this purpose it was assumed that the amount of Mo found in the screw shadow, $\approx 10^{17} \mathrm{~cm}^{-2}$, was deposited at constant rate during the total on-time of the source. The sputtering yield of this redeposited Mo due to the backstreaming ions was estimated by the sputtering yield of bulk Mo calculated by TRIM.SP of $\approx 0.01$. With these numbers, the balance of deposition and sputtering leads to a steady-state Mo coverage of the surface of $\approx 5 \%$. Hence, the influence of deposition on the sputtering by the backstreaming ions should be negligible.

Table 4 compares the results for the calculated backstreaming ion fraction obtained at 0.4 and 0.6 Pa to the RBS estimates. Estimates of the first-campaign calculations were of $2.4 \%$ compared to $2.2-2.7 \%$ for RBS. Considering the uncertainties and the simplifying assumtions of the calculation the agreement between calculation and experiment is fair in all cases.

\begin{tabular}{|c|c|c|c|}
\hline & Calc. & RBS Cu & RBS Cr \\
\hline $0.4 \mathrm{~Pa}$ & $1.4 \%$ & \multirow{2}{*}{$2.5 \%$} & \multirow{2}{*}{$0.8 \%$} \\
\cline { 1 - 2 } $0.6 \mathrm{~Pa}$ & $2.1 \%$ & & \\
\hline $1^{\text {st }}$ camp. & $2.4 \%$ & $2.2-2.7 \%$ & \\
\hline
\end{tabular}

Table 4. Comparison of backstreaming ions fractions between experiment and 1D simulation. 
On the probes located on the side walls, deposition of Cs and Mo dominated in both campaigns with no evidence of any sputtering of the $\mathrm{Cu} / \mathrm{Cr}$ strips. Furthermore, after both campaigns the $\mathrm{Cs}$ on these side wall probes appears to have reacted with water after venting the chamber after the campaigns. The resulting $\mathrm{CsOH}$ seems to have attacked the copper and to a much lesser extent chromium layers, leading to a mixing of $\mathrm{Cu} / \mathrm{Cr}, \mathrm{Mo}$, and Cs.

The result that only the sputter probes mounted at the cone showed signs of erosion, but not those mounted on the side walls, supports the conclusion that the cone was the remaining copper source in MANITU. Recently, the cone - like already all other internal surfaces of the source except the side walls - was also coated by molybdenum, and no copper traces are found with spectroscopy since; this provides further evidence.

\section{Conclusion}

The flux and the energy distribution function of backstreaming positive ions in a high power negative ion source were estimated by a simple one-dimensional calculation. The calculated results were checked for consistency with the erosion found on sputter probes that were exposed to the backstreaming ion flux. The probes consisted of thin strips of copper and chromium on a lighter metal as substrate. The probes were installed at the cone-shaped transition from the driver to the source body ("cone") and at the side walls of the source body. Erosion was quantified by Rutherford backscattering analysis before and after exposure.

Generally fair agreement was found between calculation and experiment. The calculation predicted a value of the backstreaming ion flux between 1.4 and $2.1 \%$ of the flux of negative ions extracted from the source. With an energy-averaged sputtering yield obtained using the calculated energy distribution of the backstreaming ions, the campaign-averaged backstreaming ion fraction could be calculated from the erosion found on the sputter probes. These experimental values range between 0.8 and $2.5 \%$, which is in fair agreement with the 1D simulation results.

Erosion was only observed on the probes installed at the cone, not on those installed at the side walls. This observation corroborates the assumption that the erosion is indeed caused by backstreaming ions and that the cone was the remaining source of copper impurities in the plasma. 
A study on backstreaming positive ions on a high power negative ion source for fusion 14

[1] Speth E. et al. Reports on Progress in Physics, 52:57, 1989.

[2] Hemsworth R. S. et al. Review of Scientific Instruments, 79:02C109, 2008.

[3] Fubiani G., de Esch H. P. L., Simonin A., and Hemsworth R. S. Physical Review Special Topics $A B, 11: 014202,2008$.

[4] de Esch H. P. L., Svensson L. and Riz D. APS Conference Proceedings, 1097:309, 2009.

[5] Umeda N. et al. Fusion Engineering and Design, 84:1875, 2009.

[6] Fantz U. et al. Plasma Physics and Controlled Fusion, 49:B563, 2007.

[7] McNeely P., Dudin S. V., Christ-Koch S., Fantz U., and the NNBI Team. Plasma Sources Science and Technology, 18:014011, 2009.

[8] Kraus W. et al. AIP Conference Proceeding, 1097:275, 2009.

[9] Kraus W., Fantz U., and Franzen P. Review of Scientific Instruments, 81:02B110, 2010.

[10] Franzen P. et al. Fusion Engeneering and Design, 82:407, 2007.

[11] Fantz U. et al. AIP Conference Proceeding, 1097:265, 2009.

[12] Heinemann B. et al. Fusion Engeneering and Design, 84:915, 2009.

[13] Franzen P. et al. AIP Conference Proceeding, 1097:451, 2009.

[14] Franzen P. et al. Nuclear Fusion, 47:264, 2007.

[15] Speth E. et al. Nuclear Fusion, 46:S220, 2006.

[16] Tawara H., Itikawa Y., Nishimura H., and Yoshino M. Journal of Physical and Chemical Reference Data, 19:617, 1990.

[17] Tabata T. and Shirai T. Atomic Data and Nuclear Data Tables, 76:1, 2000.

[18] Fogel Y. M., Koval A. G., Levchenko Y. Z., and Khodyachikh A. F. Soviet. Phys. JETP, 12:384, 1961.

[19] Krylov A. and Hemsworth R. S. Fusion Engeneering and Design, 81:2239, 2006.

[20] Fantz U. et al. Nuclear Fusion, 46:S297, 2006.

[21] Fantz U. et al. Nuclear Fusion, 49:125007, 2009.

[22] Lorentz A., Fantz U., and Franzen P. Technical Report IPP, 2006.

[23] Surrey E. Nuclear Fusion, 46:S360, 2006.

[24] Eckstein W. Computer simulation of ion-solid interactions. Springer Series in Materials Science. Springer Verlag, Berlin and Heidelberg, 1 edition, 1991.

[25] Eckstein W. Sputtering yields. In R. Behrisch and W. Eckstein, editors, Sputtering by Particle Bombardment IV, volume 110 of Topics in Applied Physics, chapter Sputtering Yields, page 33. Springer Verlag, Berlin, 2007.

[26] Bodhansky J., Roth J., and Brossa F. Journal of Nuclear Materials, 85-6:1145, 1979. 